\title{
Diseño de un sistema fotovoltaico autónomo para el uso en áreas recreativas en la zona costera
}

\author{
Design of an autonomous photovoltaic system for use in recreational areas in the \\ coastal zone
}

CARRILLO-ORTEGA, Elvis $\dagger^{*}$, MEDINO-TREVIÑO, Marco Antonio, PEREZ-LUNA, Jorge Daniel y CASTILLO-RAMIREZ, Carlos Eduardo

Universidad Tecnológica de Altamira

ID 1 ${ }^{\text {er }}$ Autor: Elvis, Carrillo-Ortega / ORC ID: 0000-0001-6328-6083, CVU CONACYT ID: 982019

ID $1^{\text {er }}$ Coautor: Marco Antonio, Merino-Treviño / ORC ID: 0000-0002-7542-5746, CVU CONACYT ID: 295355

ID $2^{\text {do }}$ Coautor: Jorge Daniel, Perez-Luna / ORC ID: 0000-0002-7834-1441, CVU CONACYT ID: 1018106

ID $3^{\text {er }}$ Coautor: Carlos Eduardo, Castillo-Ramírez / ORC ID: 0000-0003-2378-4226, CVU CONACYT ID: 1001435

DOI: $10.35429 /$ JEE.2019.11.3.22.27

Recibido Septiembre, 20, 2019; Aceptado Diciembre 20, 2019

\section{Resumen}

Estación fotovoltaica creada sobre un armazón de metal y madera aprovechando la geometría de una sombrilla convencional, fabricada en malla-sombra de las que son utilizadas en las playas o terrazas proyectando su sombra con el propósito de evitar una insolación a los usuarios. Se desarrolló un elemento multifuncional, que combina ecología y tecnología, el cual emplea tecnología fotovoltaica para dar vida a gadgets y luminarias led integradas. Los 2 módulos fotovoltaicos situados sobre la estructura no solo sirven para genera electricidad sino también para brindar protección de la radiación solar. Para el desarrollo de este proyecto han colaborado ingenieros eléctricos, electrónicos, en energías y distintos expertos en la materia. Esta estación está integrada por $50 \mathrm{Wp}$ y 1 batería de ion-litio que hacen posible la producción y el almacenaje de energía eléctrica proveniente del recurso solar. Gracias a este sistema, el abastecimiento de electricidad está garantizado incluso cuando existe un bajo nivel de radiación solar. La capacidad de recarga de esta instalación es de hasta $12000 \mathrm{mAh}$ a la vez una disponibilidad durante las 24 horas del día.

Fotovoltaica, Radiación, Gadgets

\begin{abstract}
Photovoltaic station created on a frame of metal and wood taking advantage of the conventional umbrella geometry, made of mesh-shadow of those used on beaches or terraces, projecting its shadow in order to avoid sunstroke to users. A multifunctional element was developed; combining ecology and technology, which uses photovoltaic technology to provide charge to gadgets and integrated LED luminaires. The 2 photovoltaic modules located on the structure not only serve to generate electricity but also to provide protection from solar radiation. For the development of this project, electrical, electronics, energy engineers and various experts in the field have collaborated. This station includes $50 \mathrm{Wp}$ and 1 lithium-ion battery that makes possible the production and storage of electrical energy coming from the solar resource. Thanks to this system, the supply of electricity is guaranteed even when there is a low level of solar radiation. The recharge capacity of this installation is up to $12000 \mathrm{mAh}$ at the same time an availability 24 hours a day.
\end{abstract}

Photovoltaics, Radiation, Gadget

Citación: CARRILLO-ORTEGA, Elvis, MEDINO-TREVIÑO, Marco Antonio, PEREZ-LUNA, Jorge Daniel y CASTILLORAMIREZ, Carlos Eduardo. Diseño de un sistema fotovoltaico autónomo para el uso en áreas recreativas en la zona costera. Revista de Ingeniería Eléctrica. 2019. 3-11: 22-27

$\dagger$ Investigador contribuyendo como primer Autor.

*Correspondencia al Autor correo electrónico: (ecarrillo@utaltamira.edu.mx) 


\section{Introducción}

La contaminación es uno de los problemas más grandes que aquejan a la humanidad, no solo por sus devastadores efectos, sino porque llegará el momento en el que ya no se podrá hacer nada al respecto. Ante tal amenaza, se hace la cuestión de por qué no utilizamos más las energías renovables, después de todo una de las principales fuentes de contaminación es la producción de energía eléctrica, haciendo uso de ellas podríamos amortiguar la escases de combustibles fósiles que se va a generar en un futuro.

Ante tales panoramas, una de las posibles soluciones sería la energía solar, después de todo. La energía solar es una de las energías más abundantes, y además de que esta se encuentra en todas partes, por otro lado es una de las energías no contaminantes al no generar emisiones de dióxido de carbono a la atmosfera, como lo es los generadores de diésel, que además de que estos suelen ser muy ruidoso, son muy voluminosos y pesados, un módulo fotovoltaico tiene la ventaja de que puede trabajar de manera independiente de la red eléctrica. (León, ENSEÑAT, J, \& MARTINEZ, C, 2007)

Para fomentar el uso de la energía solar, se propone diseñar y elaborar una palapa de playa/ sombrilla de patio, que brinde un suministro eléctrico para dispositivos móviles, y que cuente con iluminación para la noche, estará destinado a las zonas de ecoturismo altamente concurridas como lo son las playa, parques públicos y lagos.

Debido a que los módulos FV se encuentran en la estructura que se asemejara a una palapa de playa y este a su vez se encontrara en la zona costera en el área recreativa, el usuario se quita la molestia de andar cargando con un panel en todos lados, además de que, al ser instalado en una palapa de playa, este no contamina visualmente debido a que toma como base un objeto que ya se encontraba en la región. Por si fuera poco la sombrilla cuenta con iluminación para la noche. A diferencia de la sombrilla presentada por la compañía B-Part (Bpart innovacion tecnológica , 2017) que cuenta con una potencia de $12 \mathrm{~W}$, en el cual nuestro diseño contará con hasta 4 veces mayor potencia ( 2 módulos FV de $25 \mathrm{~W}$ cada uno, para una potencia de $50 \mathrm{~W}$ juntos).

\section{Justificación}

Debido a los beneficios de la energía distribuida, esta sombrilla ofrece la facilidad de brindar electricidad a dispositivos móviles en el lugar en donde lo necesitan, de esta manera ahorrando la energía que de otra manera se perdería en la transmisión y distribución (Rochín, 2014), si se realizara de la de la forma tradicional. Dicho de otro modo, la generación de energía se realiza en el lugar en donde se consume (Padilla, 2019). Al solo necesitar de la radiación solar para funcionar, esta es independiente de la red eléctrica. (CEMDA, 2017) Y su producción de energía, no contribuye a la liberación de contaminantes de efecto invernadero. Este proyecto se realizó pensando en las necesidades de hoy en día y que cumpla con ellas para seguir conviviendo o trabajar a cualquier hora y en cualquier lugar donde deseemos estar.

\section{Problema}

Existe una demanda por energía eléctrica para dispositivos móviles en las zonas costeras, debido a su alta concurrencia de parte de los usuarios y turistas, A la cual la energía solar representa una solución satisfactoria para estos usuarios debido a su practicidad y comodidad que brinda con respecto a otras alternativas para resolver este mismo problema las power banks ya que elimina la tediosa necesidad de cargar con una batería externa, además de que el tiempo de vida de un dispositivo de este tipo es corto.

Un módulo fotovoltaico instalado en la zona de palapas a la orilla de la playa sección turística privada, se perfilaría como una solución para reactivar la economía durante la noche, ya que con estos y la ayuda de una batería, podría iluminar la palapa para que los consumidores no solo permanezcan durante el día, sino que también durante la noche al aumentar la seguridad. De esta forma los renteros $\mathrm{o}$ mercaderes, puedan arrendar palapas durante parte de la noche y que no solo dispongan del día para obtener ganancias.

\section{Objetivos}

Objetivo: diseñar y construir un sistema fotovoltaico aislado, capaz de asemejarse a una palapa de playa, que sea apto para soportar la carga demandada de dispositivos móviles y disponer de iluminación durante la noche. 


\section{Objetivos específicos}

- Elaborar una estructura capaz de soportar los paneles solares, una batería que cubra el consumo durante la ausencia de irradiación solar, y toda la electrónica de control como controlador de carga y puertos USB.

- $\quad$ Identificar el tipo de panel necesario para suministrar la carga de tal manera que pueda solventar la demanda eléctrica causada por los dispositivos móviles y la iluminación led.

- Identificar y seleccionar el tipo de luminaria para el campo de aplicación, con la técnica lumen.

\section{Marco teórico}

A lo largo de los años hemos sido dependientes casi en su totalidad de los recursos fósiles, debido a su alta densidad de energía, que se traduce en que la cantidad de joule almacenada por kilogramo es de las más altas, claro que detrás de la energía nuclear, la gasolina que tiene una densidad de energía de $45 \mathrm{MJ} / \mathrm{kg}$ (Golnik, 2003), gracias a esta característica y aunado a su facilidad de transportación, la convirtieron en el motor que impulsaba las tecnologías del siglo pasado. Sin embargo, no se puede dejar de lado que esta fuente de energía produce gases de efecto invernadero, los cuales dañan al medio ambiente y favorecen el calentamiento global, y aun si consiguiéramos resolver este inconveniente, dicho combustible se tiene previsto que se acabe en un futuro próximo debido a su naturaleza no renovable.

Las energías no renovables se le consideran a todas aquellas energías que no se pueden renovar en un periodo de tiempo considerable. Dicho esto, se deja en claro que las energías renovables son las que se renuevan en un tiempo corto. Sin embargo al indagar en el ámbito renovable es imposible no toparse con otro término usado con mucha frecuencia que es las energías limpias, y aunque ambas son propias de la energía solar, es necesario aclarar las diferencias entre ambos términos. Con la referencia anterior sobre las energías renovables podemos creer que esta se encuentra en el área de las energías limpias, o incluso que es un sinónimo, aunque esto caería en la equivocación.
Las energías limpias se consideran a todas esas fuentes de energías que no generan como residuo de la producción de energía el dióxido de carbono.

\section{Metodología de investigación}

Las medidas de las cargas, como voltaje corriente se tomaron a partir de medidas realizadas con un multímetro. Las medidas de irradiación de la toma se tomaron de la página inventario nacional de energías limpias. (INEL, 2019). La tecnología empleada es factible en términos técnicos debido a la disponibilidad de materia prima en la zona de aplicación, y a la abundante radiación en la zona.

\section{Resultados}

\begin{tabular}{|l|r|r|r|r|}
\hline \multicolumn{1}{|c}{ Carga } & \multicolumn{2}{c}{$\mathbf{N}^{\circ}$} & \multicolumn{1}{c|}{$\mathbf{P}(\mathbf{W})$} & \multicolumn{1}{c|}{$\begin{array}{c}\text { Uso } \\
\text { (hrs) }\end{array}$} \\
\hline $\begin{array}{l}\text { Puertos } \\
\text { USB }\end{array}$ & 2 & 5 & 4 & 40 \\
\hline Luces led & 8 & 0.32 & 6 & 15.6 \\
\hline
\end{tabular}

Tabla 1 Censo de carga

\begin{tabular}{|l|r|}
\hline \multicolumn{1}{|c|}{ Mes } & Irradiación Wh/m²/día \\
\hline Enero & $3,555.00$ \\
\hline Febrero & $4,421.00$ \\
\hline Marzo & $5,495.00$ \\
\hline Abril & $6,281.00$ \\
\hline Mayo & $6,760.00$ \\
\hline Junio & $6,780.00$ \\
\hline Julio & $6,510.00$ \\
\hline Agosto & $6,383.00$ \\
\hline Septiembre & $5,382.00$ \\
\hline Octubre & $4,871.00$ \\
\hline Noviembre & $3,979.00$ \\
\hline Diciembre & $3,455.00$ \\
\hline
\end{tabular}

Tabla 2 irradiación en la zona costera

\section{Cálculo luminarias}

Para la obtención de la cantidad y tipo de luces que se utilizará, primero se debe establecer los lúmenes que se necesitarán. En el caso de una sombrilla/ palapa de playa que se encontrara en zonas al aire libre, exteriores, se obtuvo siguiendo la norma NOM-025-STPS-2008 (JAVIER LOZANO ALARCON, 2008)

Niveles de iluminación que debe cumplir para cada tipo de tarea: 


\begin{tabular}{|c|c|c|}
\hline $\begin{array}{c}\text { Tarea Visual } \\
\text { del Puesto de } \\
\text { Trabajo }\end{array}$ & Área de Trabajo & $\begin{array}{c}\text { Niveles } \\
\text { Mínimos de } \\
\text { Iluminación } \\
\text { (Luxes) }\end{array}$ \\
\hline $\begin{array}{l}\text { En exteriores: } \\
\text { distinguir el } \\
\text { área de } \\
\text { tránsito, } \\
\text { desplazarse } \\
\text { caminando, } \\
\text { vigilancia, } \\
\text { movimiento } \\
\text { de vehículos. }\end{array}$ & $\begin{array}{l}\text { Exteriores generales: } \\
\text { patios } \\
\text { estacionamientos. }\end{array}$ & 20 \\
\hline $\begin{array}{l}\text { En interiores: } \\
\text { distinguir el } \\
\text { área de } \\
\text { tránsito, } \\
\text { desplazarse } \\
\text { caminando, } \\
\text { vigilancia, } \\
\text { movimiento } \\
\text { de vehículos. }\end{array}$ & $\begin{array}{l}\text { Interiores generales: } \\
\text { almacenes de poco } \\
\text { movimiento, pasillos. } \\
\text { Escaleras, } \\
\text { estacionamientos } \\
\text { cubiertos, labores en } \\
\text { minas subterráneas, } \\
\text { iluminación de } \\
\text { emergencia. }\end{array}$ & 50 \\
\hline
\end{tabular}

Tabla 3 Niveles Mínimos de iluminación NOM-025STPS-2008

Haciendo uso explícito de la norma, se debería tomar en cuenta 20 luxes como la cantidad iluminación a alcanzar. Sin embargo, al consultar en otras fuentes, se encontró que el libro "Iluminación Artificial De Las Zonas Verdes" (F., Ballester-Olmos, \& Anguís) sé a detalla los siguientes aspectos:

"La iluminación de un parque no debe alcanzar los niveles que se recomiendan para interiores, (...) en general se considera que 201ux es el valor mínimo aconsejable para todas las zonas por donde circulan personas (...).”

Siguiendo estas observaciones, la instalación no podría alcanzar los 50 luxes, por lo que se optó por tomar un valor inferior de 40 lux, que nos permitirá mantenernos en el rango de los 20 a 50 luxes si sucediera un infortunio como una lámpara fallando o la poca carga de una batería.

Para obtener los lúmenes se utilizó la técnica lumen. (Blanca Giménez, Vicente, Castilla Cabanes, Nuria, Martínez Antón, Alicia, \& Pastor Villa, Rosa María, 1995) En la ecuación 1 se muestra los lúmenes necesarios para el área que cubrirá la sombrilla:

lumen $=(40$ lux $)\left(4 m^{2}\right)=160$ lumen
Posteriormente se determinó el número de lámparas usando el mismo manual, dividiendo entre la cantidad de lámparas por modulo y el flujo luminoso de cada uno, como se muestra en la fórmula 2 :

$n \cdot{ }^{\circ}$ de lamparas $=\frac{160 \mathrm{~lm}}{(4) \times(5 \mathrm{~lm})}=8$

Se eligió este tipos de módulos led por cuestiones de practicidad, ya que en caso de que se dañara una no comprometería el resto de la iluminación, además de que la iluminación estaría mejor distribuida.

\section{Dimensionado de los módulos fotovoltaicos}

Se observa en general que debido a las condiciones ambientales y estado de la zona costera, esta cuenta con una irradiación apropiada para la instalación de módulos fotovoltaicos. Con el consumo diario, la irradiación de la zona y características de los módulos, se obtiene el número de módulos fotovoltaicos, como se muestra en la ecuación 3:

$N^{\circ} P=\frac{57.6 \mathrm{Wh} \mathrm{dia}}{(3.99)(10.1 \mathrm{~W})(0.65)}=2.1$ modulos

La corriente de cortocircuito de los módulos es de 1.4 Amp. Debido a que se va utilizar 2 módulos para cumplir la demanda, y son de una capacidad de $12 \mathrm{~V}$, al conectarlos en paralelo, tenemos la corriente que debe soportar el controlador es (ecuación 4):

$$
(1.4 \mathrm{Amp} .) *(2)=2.8 \mathrm{Amp}
$$

\section{Dimensionado de la batería}

Para el dimensionado de la capacidad de la batería dada en ampere hora, se utilizó la fórmula (Hontoria, 2015) (5):

$C_{n}=\frac{\left[\frac{(57.6 W h / d)(1.7)}{0.7}\right]}{12 v}=11.65 \mathrm{Ah}$

En donde se obtuvo el producto de la energía necesaria (dada en Wh dia) por el número de días de autonomía, posteriormente se adquirió el cociente de este resultado entre la profundidad de descarga de la batería, (en este caso 0.7). Finalmente se saca el cociente de este valor entre el voltaje de la batería. 


\section{Recomendaciones}

Debido a su naturaleza de sistema aislado, (EDUARDO, 2019) lleva consigo la desventaja que la batería tiene un costo elevado, además de los agentes contaminantes que contiene, sin embargo, en un futuro se tratara de reemplazar este componente de tecnología convencionales, por una más amigable con el ambiente como los sol las baterías de agua salada.

En la ilustración 1 se muestra el sistema fotovoltaico autónomo instalado en la UTA, compuesto por los módulo FV en la parte superior y el dispositivo de carga de los dispositivos electrónicos en el mástil.

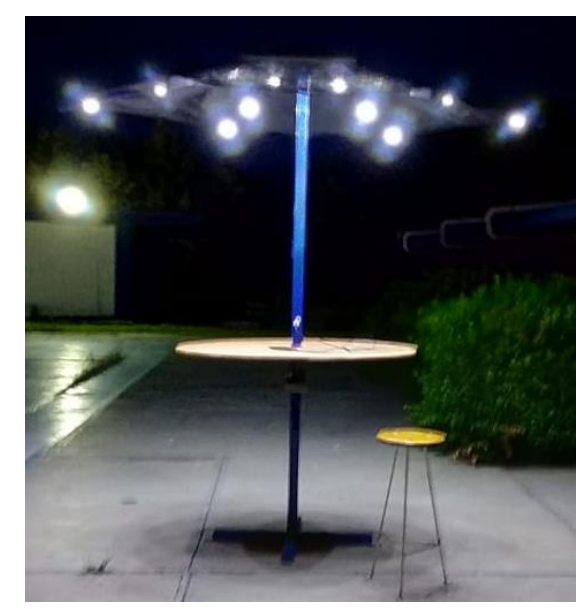

Figura 1 sombrilla fotovoltaica terminada Fuente: Elaboración Propia

\section{Conclusión}

Se dimensiono el consumo total de energía eléctrica, los modulo led y la estación de carga para dispositivos móviles, de tal forma que se obtuvo un consumo de $57 \mathrm{Wh} / \mathrm{dia}$, y en base a este valor se adquirió el dimensionado necesario de los 2 módulos de 10W, así como los valores de la batería.

Con el sistema fotovoltaico aislado de este proyecto que se encuentra en la palapa/sombrilla de playa, permite a los usuarios de gozar de las comodidades de un suministro eléctrico en la zona de recreación costera, lo cual resulta útil debido a que le ahorra la necesidad al usuario de estar cargando con baterías externas para mantener su dispositivo cargado.

\section{Referencias}

Blanca Giménez, Vicente, Castilla Cabanes, Nuria, Martínez Antón, Alicia, \& Pastor Villa, Rosa María. (1995). LUMINOTECNIA: Magnitudes Fotométricas básicas. Unidades de medida.

B-part innovacion tecnológica . (2017). b-part gadgets . Obtenido de b- part gadgets : https://bpartgadgets.com/US_en/tienda/gadgets/sombrill a-solar-de-aluminio-con-2-puertos-de-cargausb.html

CEMDA. (2017). CEMDA. 2017. Marco jurídico de las energías renovables en México. Obtenido de www.cemda.org.mx: https://www.cemda.org.mx/wpcontent/uploads/2016/06/Marcojur\%C3\% ADdico-de-las-energ\%C3\%ADasrenovables-en-M\%C3\%A9xico.final_.pdf

EDUARDO, R. P. (JUNIO de 2019). IMPLEMENTACION DE UN SISTEMA DE CARGA PARA CELULARES MEDIANTE . QUITO, ECUADOR.

F., J., Ballester-Olmos, \& Anguís. (s.f.). ILUMINACION ARTIFICIAL DE LAS ZONAS VERDES. MINISTERIO DE AGRICULTURA, PESCA Y ALIMENTACION, 5.

Golnik, A. (2003). The Physics Factbook. Obtenido de The Physics Factbook: https://hypertextbook.com/facts/2003/ArthurGo lnik.shtml

Hontoria, J. A. (2015). Dimensionado de sistemas fotovoltaicos autónomos. Obtenido de CIEMAT.

INEL. (2019). inventario nacional de energias limpias . Obtenido de https://dgel.energia.gob.mx/INEL/mapa.html?la $\mathrm{ng}=\mathrm{es}$

JAVIER LOZANO ALARCON. (30 de diciembre de 2008). NORMA Oficial Mexicana NOM-025-STPS-2008, Condiciones de iluminación en los centros de trabajo. México: DIARIO OFICIAL. 
León, I. d., ENSEÑAT, J, \& MARTINEZ, C. (2007). www.iccl.es. Obtenido de www.iccl.es: http://energia.jcyl.es/web/jcyl/binarios/247/383/ Guia\%20Solar_Completa_reducida_2.pdf?blob header=application $\% 2$ Fpdf $\% 3$ Bcharset $\% 3 D U T$ F-8\&blobheadername1=CacheControl\&blobheadername2 $=$ Expires\&blobhead ername $3=$ Site $\&$ blobheadervalue $1=$ nostore $\% 2$ Cno-cache $\% 2$ Cmust-rev

Padilla, I. A. (10 de marzo de 2019). Energia hoy Obtenido

de https://energiahoy.com/2019/03/10/generaciondistribuida-y-financiamiento/

Rochín, V. R. (3 de abril de 2014). Termodinámica. 